\title{
Knowledge, attitudes and practice of healthcare providers regarding contraceptive use in adolescence in Mahalapye, Botswana
}

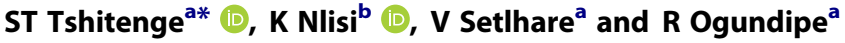 \\ ${ }^{a}$ Family Medicine and PH, University of Botswana, Gaborone, Botswana \\ ${ }^{b}$ Botswana Ministry of Health and Welfare, Mahalapye, Botswana \\ ${ }^{*}$ Corresponding author, email: stephotshitenge@gmail.com
}

Introduction: Adolescent pregnancy is a global public health problem, for which healthcare providers (HCPs) play a critical role to prevent unintended pregnancy. This study investigated the knowledge, attitude and practice (KAP) of HCPs towards the use of contraceptives in adolescents.

Results: Of the 101 eligible for the study, 79.2\% HCPs from the selected clinics and hospital responded.

The majority (91.2\%) of respondents felt confident to explain to adolescents how to use old contraceptive methods such as oral contraceptives or IUCD, less than half of the respondents $(41.3 \%)$ were confident to explain how to use new contraceptive methods such as transdermal contraceptive patches or vaginal rings. Medical doctors felt more confident to prescribe new contraceptive methods compared with nurses, both vaginal rings ( $p$-value $=0.0006)$ and transdermal contraceptive patches $(p$-value $=0.0003)$. More than two-thirds of the respondents disagreed that beliefs influenced their ability to offer contraceptive services to adolescents, half of the respondents strongly disagreed that it was morally wrong for adolescents to use contraceptives. Although three-quarters of respondents strongly agreed (median =5, [IQR 5-6]) that they were comfortable with prescribing contraceptives to adolescents, only $23 \%$ of the respondents very much prescribed or always prescribed contraceptives to adolescents.

Conclusion: Most of the HCPs prescribed contraceptives irregularly, and had limited knowledge about newer methods. To change HCPs' KAP, in addition to continuing medical education (CME), the establishment of family planning clinics for adolescents and more undergraduate contraceptive teaching for medical and nursing students could result in the increased utilisation of contraceptive services by adolescents.

Keywords: attitude, contraceptive in adolescents, healthcare providers, knowledge, Mahalapye Botswana, practice

\section{Introduction}

Adolescent pregnancy is a global public health problem; the World Health Organization estimates that 20000 adolescents under 18 years give birth every day worldwide. ${ }^{1}$ Although Botswana recently passed the Penal Code Amendment Bill raising the age of consent for sex from 16 years to 18 years, in April $2018,19.1 \%$ of girls in Botswana still have their first sexual intercourse before the age of 13 years. ${ }^{2,3}$ In 2011 , Botswana had about 453 students who dropped out of school because of unintended pregnancy with $9 \%$ of them being very young primary school aged students. ${ }^{2}$ Contraceptives help to reduce teenage pregnancies. Oral and injectable contraceptives are readily available in Botswana health facilities, yet their usage by adolescents is still low as a United Nations Population Fund (UNFPA) report estimates. ${ }^{4}$ Healthcare providers (HCPs) along with parents and other educators are assets to prevent unintended pregnancy in the community. For HCPs to play the critical role expected of them, they require sufficient knowledge and a positive attitude towards contraceptive use in adolescents so that they prescribe contraceptives to them to prevent unintended pregnancy.

Studies had indicated that, among HCPs, there is a deficit in knowledge of contraceptive use in general patients and in adolescents in particular. For instance, a study conducted among European HCPs in 2014 found that HCPs lacked knowledge of contraceptive methods and did not follow guidelines. ${ }^{5,6} \mathrm{~A}$ study conducted in Osun State in Nigeria in 2015 indicated that less than half of HCPs knew about all the types of contraceptive methods. ${ }^{7}$
Furthermore, HCPs lack knowledge on methods that can prevent pregnancy after unprotected sex in adolescents such as emergency contraceptive (EC) methods. As an illustration, a report from Kenya in East Africa indicated that while almost all nurses knew of at least one regular contraceptive method and half of them had heard about EC, less than $3 \%$ of them spontaneously listed EC as a contraceptive method. ${ }^{8}$

The HCPs' age seems to play a role in the selection of the types of contraceptive methods to prescribe. Young HCPs were more likely to offer a intrauterine contraceptive device (IUCD) than older HCPs. ${ }^{9}$

Apart from knowledge, culture and religious beliefs also influence HCPs' attitude towards the prescription of contraceptives in adolescents. For instance, general practitioners in India were reported to be reluctant to provide contraceptives to girls under the age of 16 as it was against their cultural values. ${ }^{10,11}$ Also, a report from a study from Ibadan in Nigeria showed a cultural reluctance towards providing contraceptives to unmarried adolescents as more than half of HCPs perceived the provision of contraceptives to such clients as promoting sexual promiscuity. ${ }^{12}$ In a study conducted in Kenya and Zambia, midwives were hostile to adolescent reproductive services, but continuing education on adolescent sexuality and reproduction reversed their negative

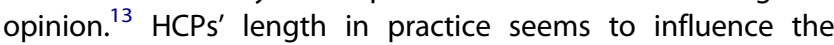
opinion and prescription of contraceptives in adolescents as recently graduated and young $\mathrm{HCPs}$ were more likely to offer contraceptives to adolescents than senior healthcare providers. ${ }^{14,15}$ 
Available studies from Botswana on the knowledge, attitude and practice (KAP) of HCPs focused on barrier contraceptives such as the female condom, or on specific populations such as those who are HIV positive. In these studies, although $95.7 \%$ of HCPs agreed that the level of knowledge on female condoms influenced their use, HCPs displayed a deficit in contraceptive knowledge and negative attitudes that discouraged HIV positive women from seeking sexual reproductive health services. ${ }^{16,17}$ Despite free sexual reproductive health services in Botswana, the country still has concerns on the usage of contraceptive by adolescents. ${ }^{4}$ There was a need to study the KAP among $\mathrm{HCPs}$ regarding contraceptive use in adolescents in such an environment.

This study investigated the KAP of HCPs towards the use of contraceptives in adolescents in the Mahalapye district health team catchments area (Mah-DHMT). It also assessed whether there was an association between the KAP of HCPs in contraceptives in adolescents and their demographic characteristics.

\section{Methods}

\section{Study design and period}

This was a cross-sectional study that used a self-administered questionnaire from November to December 2016.

\section{Setting}

Mah-DHMT's health facilities consist of one district hospital (Mahalapye District Hospital [MDH]), one primary hospital (Sefhare Primary Hospital) and 15 clinics (Airstrip clinic and Xhosa clinic are the two main clinics) and 10 health posts. All these health facilities provide contraceptive services. MahDHMT is also a training site of the University of Botswana (UB), Family Medicine Department (FM).The UB established a library that is used by medical students doing their FM rotation, residents in FM as well as the Mah-DHMT staff. The library has a variety of books, online books and medical journals. A total of 180 nurses, 15 medical officer interns, 30 medical doctors (among these nine FM residents) and 10 specialists work in Mah-DHMT. The study was carried out in MDH accident and emergency (A\&E) department, the gynaecology ward, mother and child health unit, post-natal ward, outpatient department and the youth clinic, as well as Airstrip clinic and Xhosa clinic. Statistics from Mah-DHMT indicated that these selected departments of $\mathrm{MDH}$ and clinics contributed more than $80 \%$ of contraceptive service provision in Mah-DHMT; 82 nurses and 19 medical officers worked in these departments of $\mathrm{MDH}$ and clinics.

\section{Study population}

HCPs involved in adolescent care in Mah-DHMT were targeted as they were involved in the prescription of contraceptives to adolescent patients.

\section{Sample size and selection}

The study included all 101 (82 nurses and 19 medical officers) HCP prescribers of contraceptives to adolescent clients in the selected hospital and clinics.

\section{Inclusion and exclusion criteria}

All consenting medical officers, medical interns, family medicine residents and all nurses prescribing contraceptives to adolescents were included.
We excluded those who were not contraceptive prescribers to adolescents. We also excluded specialists, doctors and nurses from the non-selected health facilities.

\section{Procedure and data collection}

A pilot study was conducted using 5 volunteer doctors and 10 contraceptive-prescribing nurses who did not belong to the health facilities selected for the study. Based on the available literature on KAP in contraceptive use in adolescents, the researcher designed a questionnaire that was assessed and corrected for its user friendliness.

Memos were distributed to all selected departments; announcements were made during doctors' and nurses' meetings. Those willing to participate in the study completed a written consent form, which was in both English and Setswana, a local language. A paper record was made available in all the selected departments and those willing to be part of the study were requested to add their names. They were then contacted physically or over the phone by the research team to sign consent forms. Once the consent form was signed, respondents were given a self-administered questionnaire to complete and submit into a secure box placed in their departments, within two days.

The questionnaire was divided into five sections: (i) background information (age, sex, education, religion and work experience), (ii) the healthcare provider's attitude to contraceptive use in adolescence, (iii) their practice, (iv) their knowledge and (v) the influence of their religion or culture in the provision of contraceptives to adolescents. The answers were on a Likert scale that ranged from 1 to $6(1=$ not sure, $2=$ strongly disagree, $3=$ disagree, $4=$ neither agree nor disagree, $5=$ agree and $6=$ strongly agree or $1=$ not at all, $2=$ a little bit, $3=$ somewhat prescribe, $4=$ quite a bit, $5=$ very much prescribe and $6=$ always prescribe). There were also dichotomous answers and respondents were asked to comment (qualitative data) at the end of each question if they felt so inclined.

\section{Analysis}

Quantitative data were summarised by reporting frequency, mean $( \pm S D)$ and median $( \pm I Q R)$ where appropriate. Tables and figures were used to summarise data. A Kruskal-Wallis $\mathrm{H}$ test and chi-square test were used to analyse the association between variables and outcome (provision of contraceptives to adolescents), where appropriate. For statistical analysis, we used R software version 3.3.1 (https://www.r-project.org/) with $\mathrm{R}$ Commander Package version 2.2-5. The level of significance of $p$-value was $<0.05$.

For qualitative data, we repeatedly read and familiarised ourselves with the written comments from respondents. We established thematic indexes using the Atlas-ti qualitative analysis software (https://atlasti.com/). Codes were applied in the thematic index; the results were charted to bring the same codes together and then interpreted all the data.

\section{Results}

\section{Profile of the respondents}

Of the 101 eligible for the study, 79.2\% (80/101) HCPs from the selected clinics and hospital responded. Two-thirds of the respondents $(50 / 80,[62.5 \%])$ were nurses, and one-third (30/ $80,[37.5 \%]$ ) were medical doctors. Forty-two respondents $(52.5 \%)$ were female and 38 respondents $(47.5 \%)$ were male; the sex ratio was 1:1. 
Table 1 displays other demographic characteristics of respondents. Three-quarters of the respondents $(72.5 \%)$ belonged either to the 20- to 29-year-old age group (34, [42.5\%]) or to the 30 - to 39 year-old age group (24, [30\%]).

Half of the respondents (40 [50\%]) were single, and the majority of the respondents (78 [97.5\%]) stated that they were Christians. Thirty-eight respondents (47.5\%) were diploma holders, 26 respondents (32.5\%) had been practising for a period of less than 5 years.

\section{Healthcare providers' knowledge of adolescence contraceptive use}

Table 2 summarises HCPs' knowledge of adolescent contraceptive use. The majority $(91.2 \%$ [73/80]) of respondents felt confident to explain how to use oral contraceptives to their adolescent clients. Less than half of the respondents $(33 / 80$ [41.3\%]) felt confident to explain how to use transdermal contraceptive patches or vaginal rings (32/80 [40\%]). Some $21 \%(17 / 80)$ of respondents were either not sure or did not know about the new methods (vaginal rings and contraceptive patches).

Medical doctors felt more confident to prescribe new contraceptive methods compared with nurses, both vaginal ring $(62.5 \%$ vs. $37.5 \%, p$-value $=0.0006)$ and contraceptive patch $(63.6 \%$ vs. $36.3 \%, p$-value $=0.0003)$.

There was a statistically significant difference in the proportion of respondents who felt more confident in prescribing an

Table 1: Demographic characteristics of the respondent healthcare providers regarding contraceptive use in adolescence in Mahalapye, Botswana, November-December 2016

\begin{tabular}{|c|c|c|}
\hline Profile of respondents & Frequency & Percentage (\%) \\
\hline \multicolumn{3}{|l|}{ Age group: } \\
\hline $20-29$ & 34 & 42.5 \\
\hline $30-39$ & 24 & 30 \\
\hline $40-49$ & 14 & 17.5 \\
\hline $50-59$ & 7 & 8.75 \\
\hline$>60$ & 1 & 1.25 \\
\hline \multicolumn{3}{|l|}{ Religion: } \\
\hline Christian & 78 & 97.5 \\
\hline Muslim & - & - \\
\hline Traditionalist & - & - \\
\hline Non-believer/other & 2 & 2.6 \\
\hline \multicolumn{3}{|l|}{ Marital status: } \\
\hline Single & 40 & 50 \\
\hline Married & 33 & 41.3 \\
\hline Living together & 6 & 6.75 \\
\hline Widow/widower & 1 & 1.25 \\
\hline \multicolumn{3}{|l|}{ Academic qualifications: } \\
\hline Diploma & 38 & 47.5 \\
\hline Degree & 37 & 46.3 \\
\hline Postgraduate & 5 & 6.3 \\
\hline \multicolumn{3}{|l|}{ Work experience } \\
\hline$<5$ & 26 & 32.5 \\
\hline $5-10$ & 26 & 32.5 \\
\hline $11-15$ & 12 & 15 \\
\hline $16-20$ & 11 & 13.8 \\
\hline$>20$ & 5 & 6.25 \\
\hline
\end{tabular}

IUCD based on marital status (51.9\% for married vs. $37.0 \%$ for single, $p$-value 0.025 ).

There was a statistically significant difference when comparing the proportion of those who answered that they used the Internet as their source of information based on their profession ( $93 \%$ for medical doctors vs. $62 \%$ for nurses, $p$-value $=0.009$ ).

Most of the respondents (70/80 [87.5\%]) used books as a source of information regarding contraceptive use; 58 (72.5\%) respondents considered themselves knowledgeable about the mechanism of action of different contraceptive methods. There were no differences in knowledge regarding the mechanism of contraceptives across the health professions $(p=0.065)$ or based on years of experience $(p=0.261)$.

Respondents suggested that the organisation of continuing medical education (CME), workshop and skills training by health institutions could be a solution to lack of the HCP knowledge and skills concerning IUCDs, new methods, side effects and the mechanism of action.

I am not trained to prescribe and insert IUCD, will need training and booklet to help with explanation.' (Respondent 78 , female nurse)

'I have recently completed my internship, have limited knowledge about contraceptive, but have attended a workshop and CME on contraceptive implants which helped a lot.' (Respondent 34, female doctor)

\section{Healthcare providers' attitude towards adolescents' contraceptive use}

The attitude of the respondents towards adolescents' contraceptive use is summarised in Table 3. Three-quarters of respondents strongly agreed (median $=5$ [IQR 5-6]) that they were comfortable with prescribing contraceptives to adolescents.

More than two-thirds of the respondents disagreed (median $=3$ [IQR 2-3]) that beliefs influenced their ability to offer contraceptive services to adolescents. Half of the respondents strongly disagreed (median $=2[\mathrm{IQR}=2-3]$ ) that it was morally wrong for adolescents to use a contraceptive.

Respondents commented that adolescents were a high-risk behaviour group at increased risk of school dropouts due to pregnancy, fatherless children, promiscuity, abortion, Caesarean section and financial problems:

'Adolescents indulge in early sexual activities, therefore they need effective contraceptive counselling; they should be allowed to make an informed decision.' (Respondent 1, 32-year-old nurse)

The advent of sex parties among adolescents was worrying for the HCPs:

'It's a disgrace that adolescents indulge in sex parties, it's unchristian.' (Respondent 2, 55-year old nurse)

\section{Healthcare providers' practice regarding adolescents' contraceptive use}

Table 4 summarises HCPs' practice regarding adolescents' contraceptive use. Three-quarters of the respondents answered 
Table 2: Healthcare providers' knowledge of adolescents' contraceptive use in Mahalapye, Botswana, November-December 2016

\begin{tabular}{|c|c|c|c|c|c|c|}
\hline \multirow{2}{*}{$\begin{array}{l}\text { Are you confident } \\
\text { to explain the } \\
\text { following to } \\
\text { adolescents? }\end{array}$} & \multicolumn{3}{|c|}{ Percentage } & \multirow{2}{*}{$\begin{array}{c}\text { Difference in the } \\
\text { proportion amongst } \\
\text { HCPs }{ }^{1} \text { who answered 'yes' } \\
\text { based on their profession, } \\
p \text {-value }\end{array}$} & \multirow{2}{*}{$\begin{array}{l}\text { Difference in the } \\
\text { proportion amongst HCPs } \\
\text { who answered 'yes' based } \\
\text { on their marital status, } p \text { - } \\
\text { value }\end{array}$} & \multirow{2}{*}{$\begin{array}{c}\text { Difference in the proportion } \\
\text { amongst HCPs who } \\
\text { answered 'yes' based on } \\
\text { their years of work } \\
\text { experience, } p \text {-value }\end{array}$} \\
\hline & Yes & No & $\begin{array}{l}\text { Not } \\
\text { sure }\end{array}$ & & & \\
\hline $\mathrm{IUCD}^{2}$ & 67.5 & 18 & 13.6 & $0.020^{*}$ & $0.025^{*}$ & 0.182 \\
\hline Vaginal ring & 40 & 36.3 & 23.8 & $0.0006^{*}$ & 0.143 & 0.376 \\
\hline Oestrogen patch & 41.3 & 35 & 23.8 & $0.0003^{*}$ & 0.012 & 0.073 \\
\hline Oral contraceptive & 91.2 & 6.25 & 2.5 & 0.366 & 0.847 & 0.506 \\
\hline $\begin{array}{l}\text { Injectable } \\
\text { contraceptive }\end{array}$ & 90 & 6.25 & 3.75 & 0.392 & 0.495 & 0.478 \\
\hline
\end{tabular}

${ }^{1}$ Health care providers; ${ }^{2}$ intrauterine contraceptive device; ${ }^{*}=p$-value statistically significant.

that they did not often prescribe contraceptive services to adolescents (median 2 [IQR 2-4]). Only 23\% (18/80) of the respondents always prescribed contraceptives to adolescents. There was no statistically significant difference in the median based on profession, marital status and work experience with a $p$-value greater than 0.05 .

Respondents stated that adolescents have rights and also commented that HCPs who lack the knowledge and necessary skills to offer contraceptive services should refer their clients to a youth clinic, or other midwives and doctors:

'Adolescents are patients and their right for autonomy should be respected.' (Respondent 3, medical doctor)

'I have limited knowledge about contraceptives, especially new ones; therefore will refer them to youth clinic or doctors.' (Respondent 10, nurse)

\section{Discussion}

This study enquired about the understanding of contraceptive prescribing by HCPs who were working within Mah-DHMT, what was their particular feeling or opinion towards the prescription of contraceptives to adolescents, and whether they were prescribing contraceptives to adolescents.

The present study revealed that, if the majority (91.2\%) of respondents felt confident to explain to adolescents how to use old contraceptive methods such as oral contraceptives or an IUCD, less than half of the respondents (41.3\%) were confident to explain how to use new contraceptive methods such as oestrogen patches or vaginal rings. Medical doctors felt more confident to prescribe new contraceptive methods compared with nurses, both vaginal rings ( $p$ value $=0.0006$ ) and transdermal contraceptive patches ( $p$ value $=0.0003$ ). The Mah-DMHT health facilities are public facilities in which, in the majority of cases, only old contraceptive methods are available. This could explain the confidence of respondents in explaining the use of old contraceptive methods to their adolescent clients. The young age of medical doctor respondents in our study could also explain their confidence in explaining the theoretical use of new contraceptive methods to their clients as they are fresh from medical training.

The study indicated that the majority $(87.5 \%)$ of respondents used books as their source of information on contraceptive

Table 3: Healthcare providers' attitude towards adolescents' contraceptive use in Mahalapye, Botswana, November-2016

\begin{tabular}{|c|c|c|c|c|}
\hline Statement & $\begin{array}{l}\text { Likert Scale } 1 \\
\text { Median } \\
(\text { IQR })^{2}\end{array}$ & $\begin{array}{l}\text { Difference in median } \\
\text { based on profession } \\
p \text {-value }\end{array}$ & $\begin{array}{l}\text { Difference in median } \\
\text { based on marital } \\
\text { status } p \text {-value }\end{array}$ & $\begin{array}{l}\text { Difference in median } \\
\text { based on years of work } \\
\text { experience } p \text {-value }\end{array}$ \\
\hline $\begin{array}{l}\text { I am comfortable with prescribing contraceptives to } \\
\text { adolescents }\end{array}$ & $5(5-6)$ & 0.873 & 0.158 & 0.518 \\
\hline $\begin{array}{l}\text { My beliefs influence the way I prescribe } \\
\text { contraceptives }\end{array}$ & $3(2-4)$ & $0.0146^{*}$ & 0.445 & 0.513 \\
\hline $\begin{array}{l}\text { In my culture, it is wrong for adolescents to use } \\
\text { contraceptives }\end{array}$ & $3(2-3)$ & 0.33 & 0.194 & 0.274 \\
\hline $\begin{array}{l}\text { My religion does not allow the use of } \\
\text { contraceptives by unmarried adolescents }\end{array}$ & $3(2-4)$ & 0.514 & 0.333 & 0.797 \\
\hline $\begin{array}{l}\text { It is morally wrong for adolescents to use } \\
\text { contraceptives }\end{array}$ & $2(2-3)$ & 0.117 & 0.671 & 0.422 \\
\hline $\begin{array}{l}\text { Providing contraceptives for adolescents promotes } \\
\text { sexual promiscuity and the spread of HIV/AIDS }\end{array}$ & $3(2-4)$ & 0.469 & 0.197 & 0.277 \\
\hline $\begin{array}{l}\text { It is better to tell sexually active unmarried } \\
\text { adolescents to abstain from sex when they ask for } \\
\text { contraceptives rather than give them } \\
\text { contraceptives when they request them }\end{array}$ & $3(2-3)$ & 0.116 & 0.466 & 0.305 \\
\hline $\begin{array}{l}\text { Adolescents should be given contraceptive } \\
\text { counselling before they become sexually active }\end{array}$ & $6(5-6)$ & 0.636 & 0.597 & 0.0934 \\
\hline
\end{tabular}


Table 4: Healthcare providers' practice regarding adolescents' contraceptive use in Mahalapye, Botswana, November-December 2016

\begin{tabular}{|c|c|c|c|}
\hline Statement & $\begin{array}{c}\text { Median } \\
(\mathrm{IQR})^{1}\end{array}$ & $\begin{array}{l}\text { Difference in } \\
\text { median } \\
\text { based on } \\
\text { profession, } \\
p \text {-value }\end{array}$ & $\begin{array}{c}\text { Difference in } \\
\text { median } \\
\text { based on the } \\
\text { marital status } \\
p \text {-value }\end{array}$ \\
\hline $\begin{array}{l}\text { How frequently do } \\
\text { you prescribe } \\
\text { contraceptives to } \\
\text { adolescents? }\end{array}$ & $2(2-4)$ & 0.181 & 0.051 \\
\hline $\begin{array}{l}\text { I have reported or } \\
\text { threatened to } \\
\text { report adolescents } \\
\text { to their parents if } \\
\text { they request } \\
\text { contraceptives }\end{array}$ & $1(1-1)$ & 0.880 & 0.808 \\
\hline $\begin{array}{l}\text { I have scolded } \\
\text { adolescents when } \\
\text { they wanted } \\
\text { contraceptives }\end{array}$ & $1(1-1)$ & 0.369 & 0.218 \\
\hline $\begin{array}{l}\text { I have refused to } \\
\text { prescribe } \\
\text { contraceptives to } \\
\text { adolescents before } \\
\text { marriage }\end{array}$ & $1(1-1)$ & 0.871 & 0.356 \\
\hline
\end{tabular}

${ }^{1}$ Interquartile range.

methods; medical doctors reportedly used the Internet more as their source of information on contraceptives compared with nurses $(p=0.009)$. There were no differences in knowledge concerning the mechanism of contraceptives across the health professions $(p=0.065)$ or based on years of experience $(p=0.261)$. Also, respondents suggested that the organisation of $\mathrm{CME}$, workshop and skills training may help to fill the gap in knowledge and skills in new contraceptive methods, their side effects and the mechanism of action. CME helps not only to improve knowledge, but also to reverse negative attitudes towards the prescription of contraceptives in adolescents. ${ }^{13}$ The UB library offers books and online materials on contraceptive methods that also help improve HCPs' knowledge of contraceptives in adolescents.

In the present study, more than two-thirds of the respondents disagreed that beliefs influenced their ability to offer contraceptive services to adolescents; half of the respondents strongly disagreed that it was morally wrong for adolescents to use contraceptive. Also, they commented that adolescents were a high-risk behaviour group at increased risk of school dropout due to pregnancy, fatherless children, promiscuity, abortion, Caesarean section and financial problems and they were worried by the advent of sex parties among adolescents.

These findings were in contrast to a study in Scotland in which doctors who had trained in the Indian subcontinent were significantly less likely to provide contraceptive services to females under the age of 16 years than those who had trained in the United Kingdom, owing to cultural influences. ${ }^{11}$ Also, a Nigerian study found that HCPs were informed by a local culture that did not support premarital sex $(p=0.02)^{12}$ and in a Ugandan study societal norms influenced attitudes of HCPs with most believing pre-marital sex was not allowed. ${ }^{18}$ A local study (in Botswana) indicated that HCPs discouraged the use of contraceptives in HIV-positive people as it was thought that this may lead to the spread of the disease. ${ }^{17}$ The young age of respondents in the present study could explain the liberal attitude of the majority of these respondents; globalisation and the Internet are breaking down barriers between Occidental and African youth.
The present study showed that three-quarters of the respondents answered that they did not often prescribe contraceptive services to adolescents and only $23 \%$ of the respondents always prescribed contraceptives to adolescents. Also, respondents expressed the view that adolescents have rights, and if an HCP lacks knowledge or the necessary skills to offer contraceptive services, he/she should refer his/her clients to a youth clinic or other midwives and doctors. Studies have indicated HCPs' irregular prescription of contraceptives to adolescents. ${ }^{6,19}$ This could be attributed to HCPs' lack of knowledge, health and safety concerns regarding contraceptives, and limited counselling skills. ${ }^{6,19}$

Though an anonymous questionnaire was used, there was a risk that respondents could have under- or overstated their responses leading to bias in results. The exclusion of condoms from the questionnaire could have limited our study findings as, due to the advent of HIV/AIDS, the focus has been on the use of condoms. The non-documentation of the respondents' number of children and their ages could also limit the study findings as those with adolescent children might have first-hand experience that impacted on how they offer contraceptive services to adolescents.

Future studies on the KAP of HCPs are needed locally to elucidate the underlying reasons why the rate of provision of contraceptive services in adolescents is low. The regularity of availability of contraceptive (IUCDs, vaginal ring, implants and oestrogen patches) in public health facilities needs to be determined as this may significantly decrease the risk of teenage pregnancy. There is a need for the introduction of evidencebased guidelines regarding the available contraceptive services for adolescents to assist the HCPs with different choices. CME (with certificates) and attitude transformation may significantly empower the HCPs. Training of the HCPs in the insertion of IUCDs and implants may also improve the uptake of these contraceptive methods. The introduction of family planning clinics, with improved contraceptive counselling skills for both male and female adolescents, may lead to more utilisation of contraceptive services and a decrease in medical costs. Students who are pursuing undergraduate medical education either as nurses or doctors may benefit from having a course in contraceptives with a specific focus on young people. Also, HCPs should have dedicated library time.

\section{Conclusions}

The knowledge, attitudes and practice of the HCPs affected the provision of contraceptive services to adolescents in Mah-DHMT. Most of the HCPs prescribed contraceptives irregularly and had limited knowledge of newer methods. A change in KAP of the $\mathrm{HCPs}$, in addition to CME, the establishment of family planning clinics for adolescents and more undergraduate contraceptive teaching for medical and nursing students may result in the increased utilisation of contraceptive services by adolescents.

Acknowledgement - This study was conducted in partial fulfilment of the requirements for the award of the Master of Medicine (Family Medicine) degree at the University of Botswana. The authors acknowledge the valuable contributions of the Mahalapye Health District staff who agreed to participate in this study.

Disclosure statement - No conflict of interest was reported by the authors. 
Ethical clearance statement - Ethical approval was sought and obtained from the Ministry of Health, Department of Health Research and Development, the University of Botswana Institutional Review Board (IRB) (UBR RES IRS GRAD 293) and Mahalapye District Hospital IRB (MH/DHMT/1/7/7 [21]) before embarking on the study.

\section{ORCID}

ST Tshitenge (D) http://orcid.org/0000-0002-7646-6443

K Nlisi (i) http://orcid.org/0000-0003-0406-5808

\section{References}

1. Goyal $M$, Zhao $H$, Mollen $C$. Exploring emergency contraceptive knowledge, prescription practices, and barriers to prescription for adolescents in the emergency department. Pediatrics. 2009;123 (3):765-70. http://doi.org/10.1542/peds.2008-0193.

2. Southern Africa Parliamentary Support Trust. Botswana raises age of consent to 18 years. April 2018 [cited 2018 May 11]. Available from: http://www.sapst.org/botswana-raises-age-of-consent-to-18-years/

3. The Botswana Youth Risk Behavioral Surveillance Survey (BYRBSS). Fact Sheet: First Botswana Youth Risk Behavioral Surveillance Survey Among 10-19 years old Students. YEAR [cited 2018 May 11]. Available from: https://photos.state.gov/libraries/botswana/19452/ pdfs/First\%20Botswana\%20Youth\%20Risk\%20Behavioral\%20Survei Ilance\%20Survey.pdf

4. UNFPA Botswana-Teenage pregnancy on the rise. 2013 [cited 2016 Jan 26]. Available from: http://countryoffice.unfpa.org/botswana/ 2013/12/06/8624/teenage_pregnancy_on_the_rise/

5. Yam EA, Gordon-Strachan G, Mclntyre G, et al. Jamaican and Barbadian health care providers' knowledge, attitudes and practices regarding emergency contraceptive pills. Int Fam Plan Perspect. 2007;33(4):160-7. http://doi.org/10.1363/ifpp.33.160.07.

6. Bratlie M, Aarvold T, Skårn ES, et al. Long-acting reversible contraceptive for adolescents and young adults - a cross-sectional study of women and general practitioners in Oslo, Norway. Eur J Contracep Reprod Health Care : Off J Eur Soc Contracep. 2014;19(3):194-202. http://doi.org/10.3109/13625187.2014.903237.

7. Omishakin MYJ. Knowledge, Attitude and Practice of Family Planning among Health care Providers in Two Selected Health Centres in Osogbo Local Government, Osun State. Women's Health Gynecol. 2015;4:12-16.

8. Adekunle AO, Arowojolu A, Adedimeji AA, et al. Adolescent contraceptive: survey of attitudes and practice of health professionals. Afr J Med Med Sci. 2000;29(3-4):247-52.
9. Uzuner $A$, Unalan $P, A k m a n ~ M$, et al. Providers' knowledge of, attitude to and practice of emergency contraceptive. The European Journal of Contraceptive \& Reproductive Health Care. Off J Eur Soc Contracep. 2005;10(1):43-50. http://doi.org/10.1080/13625180500035025.

10. Wong LP, Atefi N, Majid HA, et al. Prevalence of pregnancy experiences and contraceptive knowledge among single adults in a low socioeconomic suburban community in Kuala Lumpur, Malaysia. BMC Public Health. 2014;14(3):1. http://doi.org/10.1186/1471-245814-S3-S1.

11. Garside R, Ayres R, Owen MR, et al. General practitioners' attitudes to sexual activity in under-sixteens. J Roy Soc Med. 2000;93(11):563-4. [cited 2016 Jan 26]. Available from: http://www.ncbi.nlm.nih.gov/ pubmed/11714000. doi:10.1177/014107680009301103

12. Ahanonu E L. Attitudes of Health Care Providers towards Providing Contraceptives for Unmarried Adolescents in Ibadan, Nigeria. J Fam Reprod Health. 2014;8(1):33-40.

13. Warenius LU, Faxelid EA, Chishimba PN, et al. Nurse-midwives' attitudes towards adolescent sexual and reproductive health needs in Kenya and Zambia. Reprod Health Matter. 2006;14(27):119-28. http://doi.org/10.1016/S0968-8080(06)27242-2.

14. Vaaler ML, Kalanges LK, Fonseca VP, et al. Urban-rural differences in attitudes and practices toward long-acting reversible contraceptives among family planning providers in Texas. Women Health Iss: Off Publ Jacobs Inst Women Health. 2012;22(2):e157-62. Avilable from: doi.org/10.1016/j.whi.2011.11.004.

15. Chung-Park M. Emergency contraceptive knowledge, attitudes, practices, and barriers among providers at a military treatment facility. Mil Med. 2008;173(3):305-12.

16. Mashanda-Tafaune B, Monareng LV. Perception and attitude of health care workers towards the use of a female condom in Gaborone, Botswana. Health SA Gesondheid. 2016;21:162-170. http://doi.org/10.1016/j.hsag.2015.12.002.

17. Schaan MM, Taylor M, Puvimanasinghe J, et al. Sexual and reproductive health needs of HIV-positive women in Botswana - a study of health care worker's views. AIDS Care. 2012;24(9):1120-5. http://doi. org/10.1080/09540121.2012.672814.

18. Paul $M$, Näsström SB, Klingberg-Allvin $M$, et al. Health care providers balancing norms and practice: challenges and opportunities in providing contraceptive counselling to young people in Uganda - a qualitative study. Global Health Action. 2016;9:30283, http://doi. org/10.3402/GHA.V9.30283.

19. Wood K, Jewkes R. Blood blockages and scolding nurses: barriers to adolescent contraceptive use in South Africa. Reprod Health Matter. 2006;14(27):109-18. http://doi.org/10.1016/S0968-8080(06)27231-8.

Received: 2-02-2018 Accepted: 10-07-2018 